\title{
Stolperfallen, Missverständnisse, Handlungsempfehlungen und Erfolgsfaktoren
}

Im Folgenden soll zur Veranschaulichung möglicher Stolperfallen und Missverständnissen in Form einer zusammenfassenden Checkliste Handlungsempfehlungen ableitet werden, die letztendlich zu einer erfolgreichen Umsetzung bzw. Einführung von EDI führen sollen.

Dies soll exemplarisch am Beispiel für E-Invoicing für eine konkrete Anwendung nachfolgend dargestellt werden. Folgende Eckpunkte sollten bei der Umstellung grundsätzlich beachtet werden:

\section{Checkliste: Eckpunkte am Beispiel der Umstellung auf E-Invoicing}

- Wie viele Rechnungen werden pro Monat bzw. pro Jahr erstellt?

- Wird ein WWS für die Rechnungserstellung und den -empfang eingesetzt?

- Welche Schnittstellen aus dem und in das WWS bestehen, um die vollen Effizienzvorteile im Sinne einer automatisierten Weiterverarbeitbarkeit der Rechnungsdaten auszuschöpfen?

- Welche kundenseitigen Datenformatvorgaben müssen berücksichtigt werden, welche Vielfalt ist hier für unterschiedliche Kunden zu berücksichtigen?

- Welche (sicheren) Übertragungs- bzw. Kommunikationswege bestehen bzw. können genutzt werden?

- Welche strategischen Vorteile sollen durch die Einführung realisiert werden (z. B. Kundenbindung, Optimierung des Cash-to-Cash-Cycles) und kann ein Wertbeitrag über die reine Prozessoptimierung hinaus realisiert werden?

- Informieren Sie sich über Grund- und Zusatzleistungen von Softwareanbietern. 